\title{
Audiology
}

\section{Nasal autoinflation devices for middle ear disease in cleft palate children: are they effective?}

\author{
Dispositivi per ginnastica tubarica: efficacia nella patologia dell'orecchio medio \\ in palatoschisi
}

\author{
Cecilia Rosso, Antonia Pisani, Elisa Stefanoni, Carlotta Pipolo, Giovanni Felisati, Alberto Maria Saibene \\ Department of Otorhinolaryngology, Santi Paolo e Carlo Hospital, Department of Health Sciences, Università degli Studi di Milano, \\ Milan, Italy
}

\section{SUMMARY}

Objective. Cleft lip palate (CLP) and cleft palate (CP) patients have a higher incidence of otitis media with effusion (OME) and conductive hearing problems. This article aims to evaluate the effectiveness of a 6-month course of self-administered autoinflation therapy in paediatric CP/CLP patients in terms of conductive hearing loss (CHL) and OME prevalence.

Methods. Fifty-one patients with surgically corrected CP/CLP and diagnosis of OME received indication to 6-months autoinflation therapy with an Otovent ${ }^{\circledR}$ device. Clinical evaluation, tympanogram and pure tone audiometry were carried out at the time of prescription (T0), at the end of treatment (T1) and at 6-month follow-up (T2). Patients were divided in 2 groups based on therapeutic compliance ( 29 compliant children, group A, vs 22 noncompliant children, group B).

Results. Case series showed better audiological results and tympanometries at both time points $(\mathrm{p}<0.001)$. Group A showed better outcomes at tympanograms and at each frequency, but were statistically significant only in terms of CHL at 250 and $1000 \mathrm{~Hz}$ frequencies at T1 (respectively 0.024 and 0.012 ).

Conclusions. Nasal autoinflation therapy accelerates improvement of OME and hearing thresholds at short-/mid-term, leading to an earlier improved hearing performance.

KEY WORDS: cleft, OME, nasal autoinflation, otovent, hearing loss

\section{RIASSUNTO}

Obiettivo. I pazienti con labiopalatoschisi (CLP) e palatoschisi (CP) hanno una maggiore incidenza di deficit uditivi conduttivi e di otite media con versamento (OME). Questo articolo valuta l'efficacia di un ciclo di 6 mesi di terapia di autoinsufflazioni con dispositivo Otovent in pazienti CP/CLP in termini di ipoacusia conduttiva $(C H L)$ e prevalenza di OME. Metodi. Cinquantuno pazienti CP/CLP con diagnosi di OME hanno eseguito terapia domiciliare di autoinsufflazione di 6 mesi con il dispositivo Otovent ${ }^{\circledR}$. Valutazione clinica, timpanogramma ed esame audiometrico tonale sono stati effettuati al momento della prescrizione, alla fine del trattamento e al follow-up post-trattamento di 6 mesi. I pazienti sono stati divisi in 2 gruppi in base alla compliance terapeutica (29 pazienti gruppo A vs 22 gruppo B).

Risultati. L'intero gruppo di pazienti ha mostrato miglioramenti audiologici e timpanometrici a T1 e T2 ( $p<0,001)$. Il Gruppo A ha mostrato risultati migliori ad ogni end-point, statisticamente significativi solo per CHL alle frequenze 250 e $1000 \mathrm{~Hz}$ a Tl (rispettivamente 0,024 e 0,012).

Conclusioni. La terapia con Otovent accelera il miglioramento dei pazienti CP/CLP con disfunzioni dell'orecchio medio in termini di risultati impedenzometrici e CHL a brevemedio termine.

PAROLE CHIAVE: palatoschisi, otite media effusiva, deficit uditivo trasmissivo, autoinsufflazioni nasale, Otovent ${ }^{\circledR}$
Received: November 27, 2020

Accepted: March 1, 2021

\section{Correspondence}

Cecilia Rosso

Department of Otorhinolaryngology, San Paolo

Hospital, University of Milan, Milan, Italy

E-mail: rosso.cecilia1@gmail.com

Funding

None.

Conflict of interest

The Authors declare no conflict of interest.

How to cite this article: Rosso C, Pisani A, Stefanoni E, et al. Nasal autoinflation devices for middle ear disease in cleft palate children: are they effective? Acta Otorhinolaryngol Ital 2021;41:364-370. https://doi. org/10.14639/0392-100X-N1277

(C) Società Italiana di Otorinolaringoiatria e Chirurgia Cervico-Facciale

\section{(c) (1) $(9)$}

This is an open access article distributed in accordance with the CC-BY-NC-ND (Creative Commons Attribution-NonCommercial-NoDerivatives 4.0 International) license. The article can be used by giving appropriate credit and mentioning the license, but only for non-commercial purposes and only in the original version. For further information: https:// creativecommons.org/licenses/by-nc-nd/4.0/deed.en 


\section{Introduction}

Orofacial clefts (OFC) represent a group of congenital anomalies with incomplete closure of lips and/or palate along the midline during intrauterine development. As is widely known, OFC may lead to feeding, speech, dentition and conductive hearing disorders ${ }^{1}$. Focusing on the latter, children suffering from cleft (lip) palate (CLP) have a known susceptibility to otitis media with effusion (OME), mostly due to altered muscle function of the tensor veli palatini ${ }^{2}$. Despite the lack of conclusive data, studies focusing on paediatric CLP patients report that up to $91.1 \%$ of the CLP population have at least one episode of OME during the first 2 years of life, which is significantly higher than the $19 \%$ prevalence found in non-OFC pre-school children ${ }^{3-6}$.

Even the usual decreasing trend in OME prevalence during growth, which drops to about $10 \%$ in school-age children, is less prominent in CLP children than in the normal population ${ }^{7,8}$.

During recent years, attention to hearing problems in CLP patients has increased, acknowledging that middle ear effusion and consequent hearing loss may lead to learning issues, speech development problems and even irreversible middle ear complications ${ }^{1}$. Persisting OME during childhood may indeed lead to sequelae during adolescence and adulthood, such as conductive and/or mixed hearing loss, tympanic membrane retraction and chronic otitis media ${ }^{7}$. To avoid otological complications, ear, nose and throat specialists (ENT) strive to treat OME at the earliest stage in CLP patients. Management also varies depending on severity and comorbidities and consists of both invasive and non-invasive procedures ${ }^{9}$. Grommets placement is among the most used treatments and represents an invasive albeit efficient technique ${ }^{10}$. Among non-invasive therapies, nasal balloon autoinflation (NBAI) is a well-known treatment that allows home therapy with no need for medical monitoring at each session ${ }^{11}$. After initial presentation from the ENT, only a few children require logopaedic support to properly use the device ${ }^{4}$. NBAI forces Eustachian tube opening via nasopharynx pressure increase, allowing for drainage of middle ear fluid.

Literature studies available on NBAI point towards the overall effectiveness of these devices in the paediatric population: it is now validated by systematic reviews and international consensus conferences in which, among other things, it is defined as "the only beneficial, low-risk and low-cost non-surgical therapy" 12-14.

Nevertheless, to our knowledge, no authors have validated the use of NBAI in CLP patients, a population with a high risk of OME and other middle ear diseases. This prospec- tive study aims to evaluate the results of NBAI for the treatment of OME in paediatric CLP patients.

\section{Materials and methods}

We conducted a prospective interventional study evaluating the efficacy of 6 months of home NBAI therapy in CLP children. The Internal Review Board of the Santi Paolo e Carlo Hospital reviewed and approved the research. Patients' parents or caregivers gave their informed consent to the medical procedures described in the study.

Inclusion criteria for the study were as follows:

- personal history of surgically treated cleft-lip palate (CLP), cleft palate (CP), or submucosal palate cleft (SPC);

- age between 3 and 13 years old;

- diagnosis of OME defined as type B tympanometry with evidence of middle ear effusion at otoendoscopy at time of enrollment (at least one ear);

Exclusion criteria for the study were as follows:

- incomplete cleft surgical correction;

- other otological comorbidities: sensorineural hearing loss, tympanic membrane perforation, acute otitis media, or upper respiratory tract infections at the time of evaluation, cholesteatoma;

- CP/CLP/SPC in the context of other syndromes (e.g. Pierre-Robin sequence);

- rapid maxillary expansion planned during NBAI treatment;

- patients lacking sufficient cooperation to perform NBAI;

- history of other ENT procedures related to OME such as myringocentesis, grommets placement and adenoidectomy preceding or concurring with NBAI;

- failure to complete at least 1 year of follow up.

Seventy-two consecutive patients with surgically corrected CLP who attended our Paediatric Otorhinolaryngological Unit in the last 2 years (1 April 2018 - 1 April 2020) for either a first otolaryngological examination or during otolaryngological follow-up fulfilled inclusion criteria.

Ten patients were excluded for evidence of otological comorbidities: 3 sensorineural hearing loss, 5 tympanic membrane perforations and 2 acute upper respiratory tract infections at the time of evaluation. Two children with concurrent syndromes were excluded. One patient under rapid maxillary expansion was excluded; 3 children underwent myringocentesis, 1 had grommet placement and 4 underwent adenoidectomy before or during NBAI.

All 51 patients included in our study had a clinical indication for NBAI domiciliary therapy with a disposable balloon device (Otovent ${ }^{\circledR}$, Lofarma, Milan, Italy) along with nasal washes and underwent the same evaluation protocol. 
The protocol consists of otoendoscopy with ear wax removal and otoendoscopy, tympanometric test and audiometric exam at each time point: baseline (T0), 6-month follow-up (T1) and at 1-year follow-up (T2). Audiometric test was performed according to the age of the patient (conditioned play audiometry with patients aged 3-6 years old and pure tone audiometry with children older than 3 years) with Piano audiometer (Inventis Biomedica, Milan, Italy). Hearing loss was defined taking into account the following frequencies with air and bone conduction: $250 \mathrm{~Hz}, 500$ $\mathrm{Hz}, 1000 \mathrm{~Hz}, 2000 \mathrm{~Hz}$ and $4000 \mathrm{~Hz}$. A threshold equal to or better than $20 \mathrm{~dB}$ was considered normal and data were recorded as air-bone gaps (CHL). Each side was evaluated, and its data recorded independently.

Tympanometric curves were evaluated with an A756 middle ear analyser (Inventis Biomedica, Milan, Italy) and were classified as type A (with a middle ear pressure between +200 and $-99 \mathrm{mmH} 2 \mathrm{O}$ ), type B (flat tympanometry) and type $\mathrm{C}$ (with a middle ear pressure between -100 and $-400 \mathrm{mmH} 2 \mathrm{O})$.

Autoinflation exercises were performed at home with the Otovent ${ }^{\circledR}$ device. After the clinical indication, ENT and logopaedic specialists explained how to use the device along with illustrative video. Preliminary complimentary logopaedic sessions were offered to all families to learn how to use the device and to improve chances of compliance.

The therapeutic protocol consisted of 3 sessions per day, 3 inflations per nostril for each session. Patients were prescribed to carry out the exercises for 6 months. Patients' caregivers were instructed to keep a daily diary evaluating compliance and the number of sessions performed.

Exercises allow tubaric rehabilitation through a nasal balloon and consist of two phases: during the inflation phase the balloon is inflated via one nostril; during the deflation phase the air flows out of the balloon and into the nostril. Both the passive inflation phase and the active deflation phase increase the pressure in the nasopharynx, opening the Eustachian tube and allowing drainage of middle ear fluid. If the applied pressure doesn't achieve the one in the middle ear, inflation can be followed immediately after by swallowing to provide additional power to the Eustachian tube opening. Patients were then divided into 2 groups based on compliance. Group A was composed of compliant children (defined as correctly performing at least $70 \%$ of prescribed exercises) and group B by non-compliant children. Statistical analysis of tympanometric and audiometric results was performed between the two groups.

Data were analysed using SPSS Version 26.0 (IBM Corporation. Armonk, NY. US). Statistical tests were twosided, and a p-value $\leq 0.05$ was considered statistically significant. Since the Kolmogorov-Smirnov normality test indicated that data concerning audiometric thresholds did not follow a normal distribution (T0 $\mathrm{p}=0.008$; $\mathrm{T} 1$ and $\mathrm{T} 2$ $\mathrm{p}<0.001$ ), we chose to use non-parametric tests for all parameters, comparing $\mathrm{T} 0, \mathrm{~T} 1$ and $\mathrm{T} 2$ results by variance analysis with Friedman Test for related samples. Audiometric and tympanometric results at each time point (T0, T1 and T2) between Group A and B were analysed with the Mann-Whitney test for unpaired samples. Descriptive statistics are therefore reported as median \pm interquartile range unless otherwise stated.

\section{Results}

Fifty-one consecutive OFC patients were evaluated. Demographic results are summarised in Table I.

Analysis of the overall case series points towards a general improvement of CLP population during follow-up. The difference between tympanometric results at T0, T1 and T2 time points appears statistically significant (Wilcoxon test, $\mathrm{p}<0.001$ ). Comparison of CHL at each frequency evaluated between T0, T1 and T2 also demonstrated to be statistically different $(\mathrm{p}<0.001)$. Pairwise comparisons reported an overall improvement of CHL thresholds at each frequency and a progressive shifting from $\mathrm{B}$ curves to $\mathrm{C}$ and $\mathrm{A}$ curves at the tympanometric test from $\mathrm{T} 0$ to $\mathrm{T} 1$ evaluation and from T0 to T2 collection. No significant difference was reported between $\mathrm{T} 1$ and $\mathrm{T} 2$ evaluation points in terms of any audiometric frequency or tympanometric results (Tab. II).

Twenty-nine children reported satisfactory compliance

Table I. Demographic data of CLP population included in the study.

\begin{tabular}{llc} 
Demographic data & No. of patients \\
Gender & Male & 18 \\
\multirow{4}{*}{ Age } & Female & 33 \\
& Median & 6 \\
Cleft type & IQR & 3 \\
& Clef palate & 20 \\
& Monolateral cleft lip-palate & 16 \\
& Bilateral cleft lip-palate & 14 \\
Logopaedic & Submucosal cleft & 1 \\
aid & Required & 16 \\
Compliance & Not required & 35 \\
& Yes & 29 \\
Critical & No & 22 \\
issues & Weak parent/child & 15 \\
& Recurrent acute otitis & 4 \\
& media & 3 \\
\hline
\end{tabular}

IQR: inter-quartile range. 
Table II. Overall significance between the 3 time points and pairwise comparison of CHL in CLP population.

\begin{tabular}{|c|c|c|c|c|c|c|c|c|c|c|}
\hline & \multicolumn{2}{|c|}{ T0 } & \multicolumn{2}{|c|}{$\mathrm{T} 1$} & \multicolumn{2}{|c|}{$\mathrm{T} 2$} & \multirow[t]{2}{*}{ Overall sign } & \multicolumn{3}{|c|}{ Pairwise comparison } \\
\hline & Group A & Group B & Group A & Group B & Group A & Group B & & T0-T1 & T0-T2 & $\mathrm{T} 1-\mathrm{T} 2$ \\
\hline $250 \mathrm{~Hz}$ & $15( \pm 10)$ & $17.5( \pm 15)$ & $10( \pm 5)$ & $15( \pm 10)$ & $7.5( \pm 15)$ & $12.5( \pm 15)$ & $<0.001$ & $<0.001$ & $<0.001$ & 0.454 \\
\hline $500 \mathrm{~Hz}$ & $17.5( \pm 15)$ & $20( \pm 10)$ & $10( \pm 10)$ & $15( \pm 13.5)$ & $5( \pm 13.75)$ & $10( \pm 15)$ & $<0.001$ & $<0.001$ & $<0.001$ & 0.881 \\
\hline $1000 \mathrm{~Hz}$ & $10( \pm 10)$ & $10( \pm 15)$ & $0( \pm 5)$ & $5( \pm 12.5)$ & $0( \pm 8.75)$ & $5( \pm 15)$ & $<0.001$ & $<0.001$ & $<0.001$ & 1 \\
\hline $2000 \mathrm{~Hz}$ & $0( \pm 10)$ & $5( \pm 15)$ & $0( \pm 3.75)$ & $0( \pm 5)$ & $0( \pm 3.75)$ & $0( \pm 5)$ & $<0.001$ & 0.050 & 0.012 & 1 \\
\hline $4000 \mathrm{~Hz}$ & $5( \pm 15)$ & $7.5( \pm 15)$ & $0( \pm 3.75)$ & $0( \pm 10)$ & $0( \pm 5)$ & $0( \pm 12.5)$ & $<0.001$ & 0.017 & 0.004 & 1 \\
\hline
\end{tabular}

Significance level is 0.05 , values of significance have been adapted in accordance to Bonferroni correction for multiple tests.

with the treatment (Group A), while 22 patients did not reach sufficient adherence to the protocol according to caregivers' daily diaries (Group B).

More specifically, group B registered a median of $17 \%$ of the entire number of exercises prescribed per month (interquartile range \pm 7 , minimum $2 \%$, maximum $30 \%$ ). Causes of non-compliance were: weak parental/patient collaboration, recurrent acute otitis media which precluded the possibility of practicing NBAI exercises, impossibility to achieve an adequate pressure change despite the efforts and recurrent acute rhinosinusitis or other upper airway infections during the treatment period.

Median age in group A was $6 \pm 4$ years, while in Group B was $5.5 \pm 2$ years.

Mann-Whitney test showed that Group A and Group B did not differ in terms of baseline characteristics (Tab. III). At T1, Group A showed better outcomes in terms of CHL at each frequency evaluated, as well as a higher shift towards $\mathrm{A}$ and $\mathrm{C}$ curves at tympanograms (Tabs. III, IV). Nevertheless, the difference appears statistically significant only at 250 and $1000 \mathrm{~Hz}$ frequencies. T2 time point evaluation shows further improvements in terms of CHL thresholds and tympanogram results although not statistically significant (see Tab. III).

Comparison between CP and CLP patients in Group A with satisfactory compliance was also carried out with the Mann-Whitney test. Analysis showed no significant differences between the 2 types of clefts at each time point (Tab. IV).

Mann-Whitney test was also used to compare CHL and tympanometric results between the two age ranges (Group I: 3-6 years old, Group II: 7-13 years old) to investigate whether age affects results: analysis revealed no significant difference between the two groups.

Sixteen children (11 Group A, 5 Group B) underwent preliminary logopedic sessions. Seven patients (5 Group A, 2 Group B) required additional treatment due to unsatisfactory results with autoinflation therapy at 1-year follow-up: 4 children underwent rapid maxillary expansion, 1 patient underwent grommet placement and 2 required hearing aid fitting. There were no children who chose to switch to surgery at $\mathrm{T} 1$.

\section{Discussion}

CLP patients have a known higher rate of hearing problems than the general population. The potential sequelae during adolescence and adult life of OME and related middle ear diseases, also in terms of cognitive development, highlights the need of treating OME at the earliest stage possible.

Table III. Pairwise comparison between Group A and Group B at each time point T0, T1 and T2 with Mann-Whitney test.

\begin{tabular}{lcccccccccc} 
& & \multicolumn{2}{c}{ T0 } & \multicolumn{2}{c}{ T1 } & \multicolumn{2}{c}{ T2 } & \multicolumn{2}{c}{ Pairwise comparison } \\
CHL & & Group A & Group B & Group A & Group B & Group A & Group B & T0 & T1 & T2 \\
Median $( \pm$ IQR $)$ & $250 \mathrm{~Hz}$ & $15( \pm 10)$ & $17.5( \pm 15)$ & $10( \pm 5)$ & $15( \pm 10)$ & $7.5( \pm 15)$ & $12.5( \pm 15)$ & 0.245 & 0.024 & 0.185 \\
& $500 \mathrm{~Hz}$ & $17.5( \pm 15)$ & $20( \pm 10)$ & $10( \pm 10)$ & $15( \pm 13.5)$ & $5( \pm 13.75)$ & $10( \pm 15)$ & 0.265 & 0.090 & 0.248 \\
& $1000 \mathrm{~Hz}$ & $10( \pm 10)$ & $10( \pm 15)$ & $0( \pm 5)$ & $5( \pm 12.5)$ & $0( \pm 8.75)$ & $5( \pm 15)$ & 0.365 & 0.012 & 0.408 \\
& $2000 \mathrm{~Hz}$ & $0( \pm 10)$ & $5( \pm 15)$ & $0( \pm 3.75)$ & $0( \pm 5)$ & $0( \pm 3.75)$ & $0( \pm 5)$ & 0.364 & 0.209 & 0.735 \\
Tympanogram & $4000 \mathrm{~Hz}$ & $5( \pm 15)$ & $7.5( \pm 15)$ & $0( \pm 3.75)$ & $0( \pm 10)$ & $0( \pm 5)$ & $0( \pm 12.5)$ & 0.431 & 0.195 & 0.994 \\
& $\mathrm{~A}$ & $1.7 \%$ & $4.5 \%$ & $20.7 \%$ & $13.6 \%$ & $37.9 \%$ & $13.6 \%$ & 0.669 & 0.565 & 0.554 \\
& $\mathrm{~B}$ & $70.7 \%$ & $79.5 \%$ & $29.3 \%$ & $38.6 \%$ & $22.4 \%$ & $43.2 \%$ & & \\
\hline
\end{tabular}

Significance level is 0.05. IQR: inter-quartile range. 
Table IV. Pairwise comparison between CP and CLP patients in Group A at each time point T0, T1 and T2 with Mann-Whitney test.

\begin{tabular}{|c|c|c|c|c|c|c|c|c|c|c|}
\hline \multirow[b]{2}{*}{$\mathrm{CHL}$} & & \multicolumn{2}{|c|}{ TO } & \multicolumn{2}{|c|}{$\mathrm{T} 1$} & \multicolumn{2}{|c|}{ T2 } & \multicolumn{3}{|c|}{ Pairwise comparison } \\
\hline & & $\mathrm{CP}$ & CLP & $\mathrm{CP}$ & CLP & $\mathrm{CP}$ & CLP & TO & $\mathrm{T} 1$ & T2 \\
\hline \multirow[t]{5}{*}{ Median ( \pm IQR) } & $250 \mathrm{~Hz}$ & $15( \pm 10)$ & $15( \pm 11.25)$ & $10( \pm 7.8)$ & $10( \pm 5)$ & $0( \pm 15)$ & $10( \pm 12.35)$ & 0.190 & 0.144 & 0.250 \\
\hline & $500 \mathrm{~Hz}$ & $15( \pm 10)$ & $20( \pm 15)$ & $10( \pm 13)$ & $10( \pm 5)$ & $59( \pm 12.81)$ & $7.5( \pm 15)$ & 0.129 & 0.101 & 0.161 \\
\hline & $1000 \mathrm{~Hz}$ & $10( \pm 5)$ & $10( \pm 10)$ & $0( \pm 5)$ & $2.5( \pm 6.25)$ & $0( \pm 7.92)$ & $5( \pm 10)$ & 0.143 & 0.458 & 0.422 \\
\hline & $2000 \mathrm{~Hz}$ & $0( \pm 10)$ & $2.5( \pm 10)$ & $0( \pm 2.13)$ & $0( \pm 5)$ & $0( \pm 2.46)$ & $0( \pm 5)$ & 0.112 & 0.210 & 0.148 \\
\hline & $4000 \mathrm{~Hz}$ & $5( \pm 15)$ & $5( \pm 15)$ & $0( \pm 2.39)$ & $0( \pm 5)$ & $0( \pm 4.57)$ & $0( \pm 5)$ & 0.105 & 0.299 & 0.1 \\
\hline \multirow[t]{3}{*}{ Tympanogram } & A & $3.4 \%$ & $0 \%$ & $17.2 \%$ & $20.6 \%$ & $37.9 \%$ & $32.4 \%$ & 0.290 & 0.444 & 0.094 \\
\hline & B & $55.2 \%$ & $73.5 \%$ & $20.7 \%$ & $32.4 \%$ & $20.7 \%$ & $20.6 \%$ & & & \\
\hline & C & $24.1 \%$ & $26.5 \%$ & $44.8 \%$ & $47.1 \%$ & $24.1 \%$ & $47.1 \%$ & & & \\
\hline
\end{tabular}

Significance level is 0.05. IQR: inter-quartile range.

Among possible therapies for OME in CLP patients, NBAI represents a low-cost and low-risk strategy, applicable to the primary care setting with no need for daily monitoring ${ }^{15}$. Its lack of invasiveness allows its use without preliminary specialistic training, even if the latter may improve compliance.

Our study shows that NBAI speeds up auditory recovery in CLP patients with OME, in terms of CHL at the end of therapy (T1), with better results than in non-compliant patients. On the other hand, at 6-month follow-up (T2), the improvement reported in both groups does not appear to be significant at all at any frequency (Tab. III). However, the Wilcoxon test on the overall case series points towards a general improvement in hearing thresholds and tympanometric curves (Tab. II). These results highlight that NBAI is an effective method to accelerate hearing improvement in the short term, across an overall gain that would be most probably reached by the patient anyway. The faster improvement in Group A is reported especially at low-mid frequencies $(250 \mathrm{~Hz}: \mathrm{p}=0.024 ; 500 \mathrm{~Hz}: \mathrm{p}=0.90 ; 1000 \mathrm{~Hz}$ : $\mathrm{p}=0.012$ ) which are those more affected by OME ${ }^{16}$. This difference appears extremely important given that learning and speech development is strictly connected to hearing, and the earlier that CLP children have satisfactory hearing, the earlier they can achieve better results comparable to those of any other child.

Accordingly, tympanometric curves showed a faster improvement of middle ear function using NBAI, with a faster shift from B curves $(70.7 \%$ Group A vs $79.5 \%$ Group B at T0) to C (50\% Group A vs $47.7 \%$ Group B) and A (20.7\% Group A vs 13.6\% Group B) at T1 (Tab. IV). T2 evaluation also confirmed a better outcome for the compliant group, but with no significant difference between groups (Tab. III). Many studies have evaluated the effectiveness of NBAI therapy for OME in young healthy patients. Williamson et al. carried out a randomised controlled study of 320 children aged 4-11 years who underwent autoinflation or simple follow-up. The group evaluated tympanograms results and earrelated measures of quality of life, demonstrating that the use of NBAI in young children with OME is feasible in primary care and effective in both aspects examined ${ }^{17}$.

According to the literature, surgery with grommet insertion appears to be the most effective treatment in persistent OME with recurrent acute otitis media (rAOM) ${ }^{1}$. Nevertheless, long waiting times for grommet insertion are not unusual and treatment ends up being delayed ${ }^{19}$. BidarianMoniri et al. undertook a crossover study on 45 patients suffering from OME and waiting for grommet surgery: patients underwent both NBAI treatment and simple followup. The device demonstrated efficiency in improving both middle-ear pressure and hearing thresholds in most children after four weeks of treatment and avoided grommet surgery in 36 children ${ }^{20}$.

Moreover, a Cochrane review by Venekamp et al. on the effectiveness of grommets in children with $\mathrm{rAOM}$ is limited to five randomised clinical trials: low to very low-quality evidence suggests that children receiving grommets are less likely to have AOM recurrences compared to those managed by active monitoring, but the effect is modest with around one fewer episode at 6 months and a less noticeable effect by 12 months. It also appears uncertain whether or not grommets are more effective than antibiotic prophylaxis ${ }^{21}$.

Some reviews have shown that grommets are beneficial to the recovery of hearing in children with cleft palate and OME. Nevertheless, Kuo et al. stated that conclusions are based on underpowered cohort studies and very-lowstrength evidence ${ }^{22}$. Ponduri also noted that there is currently insufficient evidence on which to base the clinical practice of early routine grommet placement in children with cleft palate ${ }^{23}$.

In this scenario, NBAI seems a valid option to accelerate 
clinical improvement with any invasive or delayed approach. Vennik et al. explored parent and healthcare professional views and experiences of NBAI, concluding that it is an acceptable, low-cost treatment option for children with glue ear in primary care ${ }^{15}$.

In contrast, Berkman et al. compared the benefits and harms of strategies currently in use for managing OME: based on two meta-analyses of two studies ${ }^{24,25}$, NBAI did not show improvement in tympanometry and hearing levels at more than 1 month from treatment ${ }^{26}$.

To our knowledge, no studies are available about NBAI in CLP patients suffering from OME. This paper is the first one analysing the real benefits and feasibility of NBAI in accelerating the improvement process in middle ear status and hearing levels in the short- to mid-term. The group also carried out a statistical comparison between different types of cleft (CP and CLP) revealing that there are no differences between clefts in spontaneous development of hearing and tympanometric status as well as in NBAI therapy results (Tab. IV).

The small statistical significance of compared groups may be explained by the small sample size. Moreover, it is essential to remember that CLP patients report a natural improvement of middle ear diseases and in particular in OME during growth, so they are meant to decrease the trend in OME prevalence whether they undergo therapy or not, as reported in our analysis ${ }^{7,8}$. The most obvious limitation of our work is the control group. Since it was defined a posteriori, there might be some intrinsic selection biases causing worse-performing patients to be assigned to the non-compliant group (e.g. frequency of OMA episodes). Despite this design, the limitation can be fully resolved only by further prospective study, and it should be noted that audiologic baseline characteristics are not significantly different between groups A and B. Furthermore, the behaviour in the non-compliant group was sufficiently consistent, with extremely low adherence to therapy. A more inconsistent scenario among non-compliant children would have made group B an even less strong control group for statistical purposes.

Despite these shortcomings, statistical analysis revealed that NBAI accelerates the natural improvement of hearing thresholds, thus anticipating the achievement of a satisfactory hearing, potentially increasing learning and speech development. However, we did not include a control group to compare learning curves and speech evolution between NBAI treatment and non-treated CLP patients.

NBAI has restricted indications: it is useful in the case of children older than 3 years old because younger patients would not be able to practice it satisfactorily. Acute recurrent otitis media should also be excluded to avoid certain non-adherence to the treatment ${ }^{4}$. Acute adenoiditis and rhi- nosinusitis should limit its use to avoid ascending of mucopurulent secretions towards the middle ear ${ }^{4}$. Moreover, treatment options should be taken into account together with caregivers before the prescription in order to evaluate whether the child is expected to be compliant with the treatment plan ${ }^{15}$.

Provision of educational materials and demonstration of the method are likely to promote uptake and compliance ${ }^{15}$. In fact, according to our analysis, among the 16 patients who underwent preliminary logopaedic sessions, $68.75 \%$ fell into the compliant group (Group A), while $31.25 \%$ showed non-adherence (Group B).

NBAI complications are almost exceptional. A case report about pneumocephalus and orbital emphysema associated with NBAI in an adult patient is described. Their review of the literature showed similar effects just in adult patients with skull base defects relating to cranial/sinus surgery or tumours ${ }^{27}$.

Possible future developments could include a wider case series with a case-control study.

International consensus conferences already highlight the role of nasal autoinflation as the only non-surgical treatment objectively effective for OME in the general paediatric population: other medical options (steroids, antibiotics, antihistamines, decongestants) failed to demonstrate an effect on OME resolution, while still having side-effects and unfavourable cost-effectiveness. Concerning surgery, grommets seem to be an appropriate treatment for non-cleft children who have audiological impact with their OME until the condition normalises, with a higher risk of complications than those undergoing conservative treatments, the most common of which are eardrum retraction and tympanosclerosis ${ }^{14}$. Adenoidectomy should be considered in recurrent OME, for associated nasal obstruction, or in older children ${ }^{28}$.

Hearing problems in cleft patients remain less investigated in terms of therapeutical alternatives. It would be interesting to expand those findings and to compare different types of non-invasive or invasive treatments (NBAI, rapid maxillary expansion, grommet surgery and adenoidectomy) on the CLP population and to evaluate the comparative effectiveness of treatments.

\section{Conclusions}

CLP patients are a group with a well-known higher rate of diseases, including hearing problems and OME. NBAI accelerates improvement of middle ear status and hearing thresholds at short-mid-terms (6 months) in order to achieve growing results more rapidly and comparable to those of other children in hearing, learning and speech development. 


\section{References}

1 Farronato G, Cannalire P, Martinelli G, et al. Cleft lip and/or palate: review. Minerva Stomatol 2014;63:111-126.

2 Kuo CL, Lien CF, Chu CH, et al. Otitis media with effusion in children with cleft lip and palate: a narrative review. Int J Pediatr Otorhinolaryngol 2013;77:1403-1409. https://doi.org/10.1016/j. ijporl.2013.07.015

3 Paradise JL, Rockette HE, Colborn DK, et al. Otitis media in 2253 Pittsburgh-area infants: prevalence and risk factors during the first two years of life. Pediatrics 1997;99:318-333. https://doi.org/10.1542/ peds.99.3.318

4 Zingade ND, Sanji RR. The prevalence of otological manifestations in children with cleft palate. Indian J Otolaryngol Head Neck Surg 2009;61:218-222. https://doi.org/10.1007/s12070-009-0070-0

5 Flynn T, Lohmander A, Moller C, et al. A longitudinal study of hearing and middle ear status in adolescents with cleft lip and palate. Laryngoscope 2013;123:1374-1380. https://doi.org/10.1002/lary.23839

6 Sheahan P, Miller I, Sheahan JN, et al. Incidence and outcome of middle ear disease in cleft lip and/or cleft palate. Int J Pediatr Otorhinolaryngol 2003;67:785-793. https://doi.org/10.1016/ S0165-5876(03)00098-3

7 Gordon ASD, Jean-Louis F, Morton RP. Late ear sequelae in cleft palate patients. Int J Pediatr Otorhinolaryngol 1988;15:149-156. https:// doi.org/10.1016/0165-5876(88)90066-3

8 Handžić-Ćuk J, Ćuk V, Rišavi R, et al. Hearing levels and age in cleft palate patients. Int J Pediatr Otorhinolaryngol 1996;37:227-242. https://doi.org/10.1016/0165-5876(96)01412-7

9 Rosenfeld RM, Shin JJ, Schwartz SR, et al. Clinical Practice Guideline. Otolaryngol - Head Neck Surg (United States) 2016;154(1 Suppl):S1-S41. https://doi.org/10.1177/0194599815623467

10 Sederberg-Olsen JF, Sederberg-Olsen N, Holmelund M. Grommets in otitis media: a 25-year follow up. Acta Otolaryngol 2018;138:10571060. https://doi.org/10.1080/00016489.2018.1511060

11 Principi N, Marchisio P, Esposito S. Otitis media with effusion: benefits and harms of strategies in use for treatment and prevention. Expert Rev Anti Infect Ther 2016;14:415-423. https://doi.org/10.1586/1 4787210.2016.1150781

12 Schilder AGM, Venekamp RP. Nasal balloon autoinflation can help clear middle ear effusion, improving the quality of life in schoolaged children with glue ear. Evid Based Nurs 2016;19:81. https://doi. org/10.1136/eb-2015-102268

13 Perera R, Glasziou PP, Heneghan CJ, et al. Autoinflation for hearing loss associated with otitis media with effusion. Cochrane Database Syst Rev 2013;(5):CD006285. https://doi.org/10.1002/14651858. CD006285.pub2

14 Simon F, Haggard M, Rosenfeld RM, et al. International consensus (ICON) on management of otitis media with effusion in children. Eur Ann Otorhinolaryngol Head Neck Dis 2018;135:S33-S39. https://doi. org/10.1016/j.anorl.2017.11.009

15 Vennik J, Williamson I, Eyles C, et al. Nasal balloon autoinflation for glue ear in primary care: a qualitative interview study. Br J Gen Pract 2019;69:E24-E32. https://doi.org/10.3399/bjgp18X700217
16 Kaf WA, Mohamed ES, Elshafiey H. 40-Hz sinusoidal auditory steady-state response and tone burst auditory brainstem response using a Kalman filter to determine thresholds pre- and post-myringotomy with grommet tube in children with mild, low-frequency conductive hearing loss. Am J Audiol 2016;25:41-53. https://doi. org/10.1044/2015_AJA-15-0052

17 Williamson I, Vennik J, Harnden A, et al. An open randomised study of autoinflation in 4- to 11-year-old school children with otitis media with effusion in primary care. Health Technol Assess 2015;19:1-149. https://doi.org/10.3310/hta19720

18 Atkinson H, Wallis S, Coatesworth AP. Otitis media with effusion. Postgrad Med 2015;127:381-385. https://doi.org/10.1080/00325481. 2015.1028317

19 McLaren O, Toll EC, Easto R, et al. Streamlining grommet pathways for otitis media with effusion and hearing loss in children: our experience. J Laryngol Otol 2018;132:881-884. https://doi.org/10.1017/ S0022215118001603

20 Bidarian-Moniri A, Ramos MJ, Ejnell H. Autoinflation for treatment of persistent otitis media with effusion in children: a cross-over study with a 12-month follow-up. Int J Pediatr Otorhinolaryngol 2014;78:1298-1305. https://doi.org/10.1016/j.ijporl.2014.05.015

21 Venekamp RP, Mick P, Schilder AGM, et al. Grommets (ventilation tubes) for recurrent acute otitis media in children. Cochrane Database Syst Rev 2018,5:CD012017. https://doi.org/10.1002/14651858. CD012017.pub2

22 Kuo, C. L, Tsao YH, Cheng HM, et al. Grommets for otitis media with effusion in children with cleft palate: a systematic review. Pediatrics 2014;134:983-994. https://doi.org/10.1542/peds.2014-0323

23 Ponduri, S, Bradley R, Ellis PE, et al. The management of otitis media with early routine insertion of grommets in children with cleft palate-a systematic review. Cleft Palate-Craniofacial J 2009;46:30-38. https://doi.org/10.1597/07-219.1

24 Blanshard JD, Maw AR, Bawden R. Conservative treatment of otitis media with effusion by autoinflation of the middle ear. Clin Otolaryngol Allied Sci 1993;18:188-192. https://doi. org/10.1111/j.1365-2273.1993.tb00827.x

25 Stangerup SE, Sederberg Olsen J, Balle V. Autoinflation as a treatment of secretory otitis media: a randomized controlled study. Arch Otolaryngol Neck Surg 1992;118:149-152. https://doi.org/10.1001/ archotol.1992.01880020041013

26 Berkman ND, Wallace IF, Steiner MJ, et al. Otitis media with effusion: comparative effectiveness of treatments. Rockville (MD): Agency for Healthcare Research and Quality (US); 2013 May. Report No.: 13-EHC091-EF.

27 Millington AJ, Perkins V, Salam MA. Pneumocephalus and orbital emphysema associated with the use of an autoinflation device: a unique case report and review of the literature. J Laryngol Otol 2019;133:157-160. https://doi.org/10.1017/S0022215119000070

28 Saibene AM, Rosso C, Pipolo C, et al. Endoscopic adenoidectomy: a systematic analysis of outcomes and complications in 1006 patients. Acta Otorhinolaryngol Ital 2020;40:79-86. https://doi. org/10.14639/0392-100X-N0150 